\title{
Un modelo de ecuaciones estructurales para describir las relaciones entre recursos sociales, estresores y síntomas durante los tiempos del COVID-19
}

A structural equation model to describe the relationships between
social resources, stressors and symptoms during the times of COVID-19

Recepción del artículo: 29-06-2021 | Aceptación del artículo: 01-26-2022

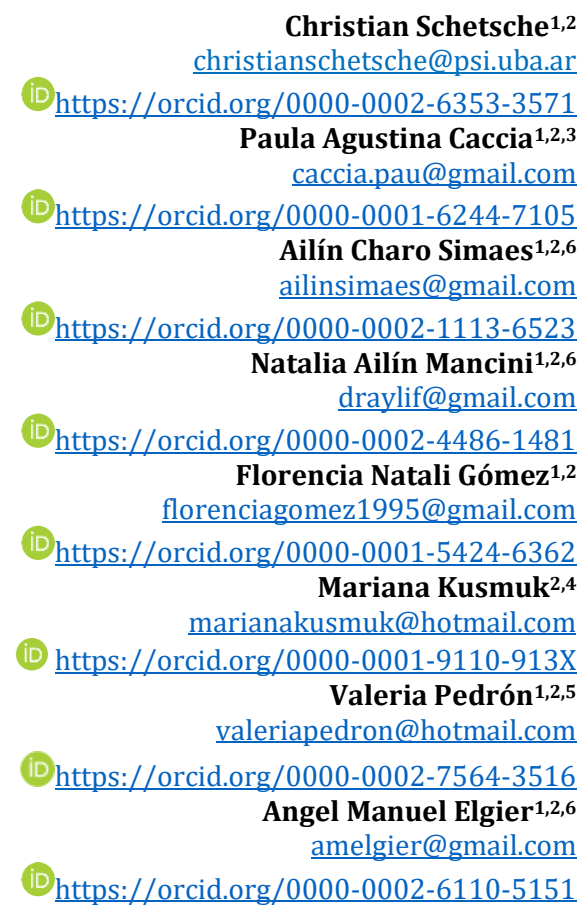

${ }^{1}$ Instituto de Investigaciones, Facultad de Psicología, Universidad de Buenos Aires. ${ }^{2}$ Laboratorio de Cognición y Políticas Públicas (CogPub).

${ }^{3}$ Facultad de Psicología y Relaciones Humanas, Universidad Abierta Interamericana (UAI).

${ }^{4}$ Escuela de Especialización y Posgrado, Área Psicología Laboral, Colegio Psicólogos Distrito XII.

5Instituto de Investigaciones, Facultad de Psicología y Psicopedagogía, Universidad del Salvador.

${ }^{6}$ Consejo Nacional de Investigaciones Científicas y Técnicas (CONICET).

Para referenciar este artículo:

Schetsche, C., Caccia, P. A., Simaes, A. C., Mancini, N. A., Gómez, F. N., Kusmuk, M., Pedrón, V. y Elgier, A. (2022). Un modelo de ecuaciones estructurales para describir las relaciones entre recursos sociales, estresores y síntomas durante los tiempos del COVID-19. ConCiencia EPG, 7(1), 124-149. https://doi.org/10.32654/CONCIENCIAEPG.7-1.8

Autora corresponsal: Schetsche Christian christianschetsche@psi.uba.ar. Buenos Aires, Argentina

\section{Resumen}

l Covid-19 no sólo es una enfermedad contagiosa, sino el causante del así llamado estrés por pandemia. Además, la cuarentena obligatoria que se impuso con el fin de disminuir el riesgo de contagio modificó la cotidianidad de las personas, diluyendo así los límites entre las rutinas familiares y laborales. Con el objetivo de determinar las relaciones entre los estresores vitales, la sintomatología depresiva y ansiosa, y los efectos amortiguadores de los recursos sociales, se utilizó un modelo de ecuaciones estructurales para realizar un análisis de sendero. Por Internet, se recolectó una muestra argentina de 637 individuos, de los cuales 182 cumplían con los requisitos de inclusión que comprendían estar con una pareja formal, estar conviviendo con hijos y encontrarse trabajando. Se determinó que la pareja como recurso consistió en el principal factor que puede reducir el riesgo de que se desarrollen síntomas depresivos y/o ansiosos. Los estresores de la pandemia y los hijos como estresores tienen las asociaciones más considerables con la ansiedad y, por otro lado, las siguientes variables tienen las correlaciones más destacadas con la depresión: la pareja como estresor, los conflictos familiatrabajo y los estresores de la pandemia. 
Se concluye que, aunque los estresores de la pandemia están significativamente correlacionados con la depresión y ansiedad, la pareja consiste en la variable clave por sus asociaciones con los síntomas analizados en el presente estudio.

Palabras Clave: Estresores vitales; Recursos sociales; Conflictos trabajo; familia y familia-trabajo; Estrés por pandemia; Depresión y ansiedad; Modelo de ecuaciones estructurales

\section{Abstract}

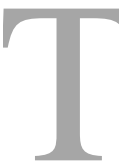

he Covid-19 is not only a contagious disease, but it is also the cause of the named "pandemic related stress". Besides, the obligatory quarantine that has been imposed to reduce the risk of contagion changed everyday nature, fading away from the limits between family and working routines. To define the relations between stressful life events, depressive and anxious symptomatology, and the damping effects of the social resources, a structural equation model has been used to carry out a path analysis. Through the internet, an Argentine sample of 637 individuals has been gathered, of which 182 fulfilled the inclusion criteria that were having a formal couple, living together with children, and being working. It has been proved that the formal couple as a resource was the principal factor that could reduce the risk of developing depressive and/or anxious symptoms. The pandemic related stress and the children as stressors have the strongest associations with anxiety. On the other hand, the following variables have the strongest correlations with depression: The couple as a stressor, the family-working conflicts, and the pandemic related stress. It is concluded that, although the pandemic related stress is significantly correlated with depression and anxiety, the couple is the key variable because of its associations with symptoms examined in the present study

Key Words: Stressful life events; Social Resources; Work-Family Conflict and Family-Work Conflict; Pandemic Related Stress; Depression and Anxiety; Structural Equation Modeling.

\section{Introducción}

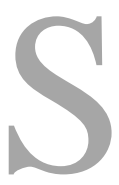

egún la Organización Mundial de la Salud (OMS), el coronavirus denominado Covid-19 es una enfermedad infecciosa que se ha descubierto recientemente (OMS, 2019). La gravedad no es únicamente el virus en sí mismo, sino la rapidez de contagio a la población (Urzúa, Vera-Villarroel, CaqueoUrízar, \& Polanco-Carrasco, 2020). Como medida preventiva, gran cantidad de países han optado por decretar cuarentena obligatoria y distanciamiento social. Esto implicó un cambio en la rutina, obligando a las personas a permanecer en sus residencias habituales y a abstenerse de concurrir a sus lugares de trabajo, generando niveles altos de preocupación, incertidumbre, miedo y angustia en la población (Johnson, Saletti-Cuesta, \& Tumas, 2020). 


\section{Los conflictos de trabajo-familia y de familia-trabajo}

Es importante tener en cuenta que este cambio de rutina, en el que los límites entre la vida familiar y laboral se han diluido de cierta manera, conlleva también la posibilidad de que surja un aumento con relación al conflicto trabajo-familia. El conflicto trabajo-familia se define como la percepción del individuo de que las responsabilidades de su trabajo y su familia interfieren entre sí (Nomaguchi, 2009), y puede tener dos direcciones: el trabajo puede interferir con la familia (conflicto trabajo-familia, CTF) y la familia puede interferir con el trabajo (conflicto familiatrabajo, CFT) (Bellavia \& Frone, 2005). Tanto el CTF como el CFT acarrean graves consecuencias para el bienestar del trabajador y de su familia (Bellavia \& Frone, 2005; Grzywacz \& Bass, 2003). Con respecto a las variables laborales que pueden influir en estos conflictos, destacan la carga horaria laboral (Fu \& Shaffer, 2001), la devoción al trabajo (Parasuraman \& Simmers, 2001) y elevados niveles de interdependencia y responsabilidad (Dierdorff \& Ellington, 2008). Con respecto a las variables familiares, se advierte que una alta participación en asuntos familiares, una elevada demanda de tiempo por parte de la familia, poco apoyo de la familia, el desacuerdo con la familia o el cónyuge, las relaciones tensas (Parasuraman \& Simmers, 2001) y la existencia y cantidad de hijos pueden aumentar los CFT (Grandey \& Cropanzano, 1999; Luk \& Shaffer, 2005).

\section{COVID-19 y el estrés por pandemia}

Además del impacto que la cuarentena obligatoria tiene sobre el entorno familiar y laboral, debe destacarse también que el distanciamiento físico, las medidas complementarias de higiene, la suspensión de actividades no prioritarias y la proliferación de información generan un nuevo contexto que establece determinadas exigencias y/o demandas de actuación a las personas. Estas demandas son potenciales estresores que representan, de acuerdo con Macías (2020), el estrés de pandemia. Ante esta situación, y las condiciones particulares que establece, el estrés de pandemia puede ser definido como un estado psicológico producido por un proceso de adaptación donde el individuo valora las demandas o exigencias del entorno, determinadas por la pandemia, como desbordantes de sus recursos para realizarlas con efectividad (Macías, 2020). Esto genera la aparición de un conjunto de síntomas o reacciones que evidencian o manifiestan el desequilibrio personal generado por el estrés que se está viviendo. Finalmente, el individuo debe llevar a cabo acciones para poder mitigar o hacer más manejable dicho estrés generado, en gran medida, por el miedo a contagiarse. Por tal motivo, estas acciones son interpretadas como estrategias de afrontamiento $y$ consisten en actos destinados a evitar el contagio (Macías, 2020).

\section{Síntomas psicológicos durante la pandemia}

Debido a los cambios generados por el Covid-19 en la cotidianidad de las personas, se han hallado efectos 
psicológicos predominando molestias de tipo ansioso y depresivo, y disminución de emociones positivas asociadas a problemas de sueño, consumo de sustancias psicoactivas, efectos físicos y percepción de la pérdida de salud (Altena et al., 2020; Huarcaya-Victoria, 2020; Ramírez-Ortiz, Castro-Quintero, Lerma-Córdoba, YelaCeballos, \& Escobar-Córdoba, 2020).

A su vez, debido a las percepciones distorsionadas de riesgo y al miedo que esto genera, aumenta la ansiedad fóbica y los comportamientos sociales negativos (Caccia, De Grandis, \& Pérez, en prensa). Estos estados y conductas pueden provocar reacciones de angustia tales como ira, temor extremo a la enfermedad incluso en personas no expuestas, trastornos específicos como trastorno de estrés postraumático y somatizaciones, entendidas como la presencia de malestares que la persona percibe relacionados con diferentes disfunciones corporales (Huremović, 2019; Shigemura, Ursano, Morganstein, Kurosawa, \& Benedek, 2020).

De esta manera, el aislamiento social y la soledad tienen la capacidad de afectar la salud mental y física de un individuo, vinculándose a consecuencias mentales y emocionales como problemas cardiacos, presión alta, deterioro cognitivo, ansiedad y depresión (Girmay \& Singh, 2019). Esto se ve acompañado por la sobre exposición a información sobre el Covid-19, aspecto que se vincula con una mayor percepción de temor irracional, síntomas paranoides y disfunciones corporales (Caccia et al., en prensa).
Asimismo, Johnson et al. (2020) afirman que, por un lado, el aislamiento por Covid-19 generó niveles altos de preocupación e incertidumbre $\mathrm{y}$, por el otro, detectaron que las personas desarrollaron un sentimiento de responsabilidad y cuidado, destacándose la valoración por la interdependencia social. Hay un reconocimiento de los sujetos como seres sociales, por lo que la empatía, la unión y la conciencia social se inscriben con un mayor valor, entendiendo que es algo indispensable para sostener las medidas sanitarias.

\section{El presente estudio}

Basado en los aumentados niveles de padecimientos psicológicos durante el tiempo de cuarentena y la emergente necesidad de determinar con exactitud sus causas, el objetivo del presente estudio comprendió la evaluación de una muestra de población adulta (1) con pareja formal, (2) que está conviviendo con hijos y (3) que está trabajando, para determinar cuáles estresores tienen las asociaciones más considerables con aumentados niveles de la sintomatología depresiva y ansiosa. Para ello, se tuvieron en cuenta las siguientes variables: estrategias que se utilizan para reducir el riesgo de contagio, estresores que surgen debido a la pandemia, la pareja como estresor, los hijos como estresores, los amigos como estresores, el trabajo como estresor, los conflictos familiares que influyen en el trabajo y los conflictos laborales que influyen en la familia. El segundo objetivo consistió en examinar cuáles recursos sociales tienen las asociaciones más considerables con reducidos niveles de los síntomas. Para la 
realización de este análisis, se consideraron las siguientes variables: la pareja como recurso, los hijos como recursos, los amigos como recursos, el trabajo como recurso. El tercer objetivo comprendió evaluar cómo se asocian los recursos entre sí, y los estresores y conflictos entre sí. Para ello, se concretó la necesidad de desarrollar un modelo de ecuaciones estructurales que no sólo incluye determinados síntomas y factores estresantes, sino también los recursos sociales, ya que éstos cumplen un rol importante al afrontar el estrés ante eventos vitales (Billings \& Moos, 1981). La Ilustración 1 representa el modelo inicial hipotetizado.

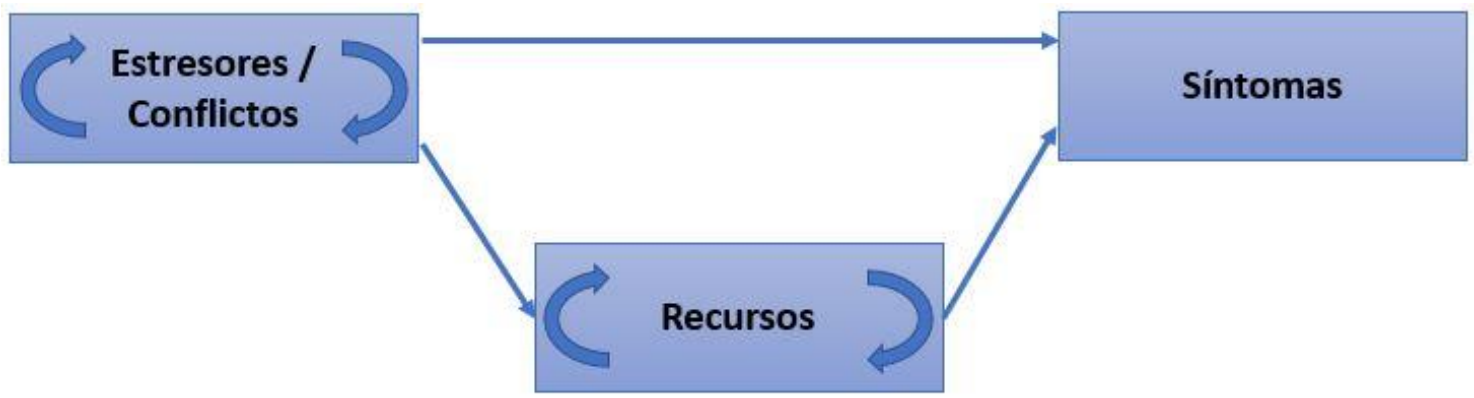

Figura 1. Modelo hipotetizado de relaciones entre estresores, recursos y síntomas

\section{Método}

Participantes: Se realizó un muestreo intencional y por bola de nieve. La muestra se recolectó entre el 14 de septiembre y el 09 de noviembre de 2020 y estuvo conformada por un total de 637 adultos (M edad $=38.14, \mathrm{DS}=13.05$, femenino $=487)$ residentes en Argentina. De éstos, 524 participantes estaban realizando actividades laborales, 443 se encontraban en una relación formal y 249 estaban viviendo con uno o más hijos.

Debido al objetivo del estudio, solamente pudieron ser incluidos aquellos individuos que cumplían con todos los requisitos establecidos: (1) encontrarse trabajando, (2) en una relación formal y (3) convivir con los hijos. De esta manera, la muestra que pudo ser analizada se redujo a un total de 182 individuos (M edad= 43.80,
DS $=8.33$, femenino= 144). El 24.2\% de estos participantes provenía de la Ciudad Autónoma de Buenos Aires, el 60.4\% de la Provincia de Buenos Aires y 15.4\% de otras provincias de Argentina.

A raíz de la escasa cantidad de participantes masculinos, no se han podido desarrollar modelos independientes por género, sino uno solo que engloba la totalidad de participantes. Para poder confirmar la validez de los resultados, se decidió realizar un análisis de invarianza factorial por género.

Instrumentos: Se utilizó el Symptom Assessment-45 Questionnaire (SA-45) de Sandín, Valiente, Chorot, Santed y Lostao (2008). El instrumento dispone de un total de 45 ítems que corresponden a nueve dimensiones: somatización, obsesióncompulsión, sensibilidad interpersonal, 
depresión, ansiedad, hostilidad, ansiedad fóbica, ideación paranoide y psicoticismo. Para la contestación, se utiliza una escala Likert de cinco puntos ( $0=$ Nada en absoluto hasta $4=$ Mucho o extremadamente) $\mathrm{y}$, en el mencionado estudio de validación en lengua española, se obtuvieron unas consistencias internas entre $.63 \leq \alpha \leq .85$. Teniendo en cuenta la extensión del presente estudio y con el fin de evitar el efecto fatiga en los participantes, se decidió utilizar solamente los factores que hacen referencia a la depresión y la ansiedad.

El inventario SISCO del Estrés de Pandemia fue desarrollado por Macías (2020) y dispone de tres factores. El primero hace referencia a los estresores que surgen durante una pandemia (por ejemplo, "Que nuestro sistema de salud colapse."), el segundo factor se refiere a determinados síntomas característicos (por ejemplo, "Falta de sueño") y el último que evalúa las estrategias que se utilizan para reducir el riesgo de contagio (por ejemplo, "Tener el menos contacto posible con las personas"). El instrumento dispone de un total de 15 ítems de los cuales cinco corresponden a cada uno de los tres factores mencionados. En su estudio de validación en el contexto argentino, (Caccia, De Grandis, \& Schetsche, en prensa) encontraron que el instrumento dispone de una estructura bi-factorial, por lo que puede ser utilizado también con un factor global que representa el estrés por pandemia. Para responder a las preguntas del inventario SISCO del Estrés de Pandemia, se utiliza una escala Likert de seis puntos que abarca los valores $0=N a d a$ hasta $5=$ Mucho. En el estudio de validación argentina, las consistencias internas alcanzaron unos valores .66 $\leq \alpha \leq .82$. Debido a la gran similitud entre las dimensiones que son evaluadas a través del $S A-45$ y los ítems correspondientes al factor síntomas, se decidió excluir el mencionado factor del presente estudio, por lo que se utilizaron solamente las dimensiones estresores y estrategias.

Life Stressors and Social Resources among Adults (LISRES-A). Adaptación argentina (Mikulic, 1998). El LISRES-A evalúa los estresores vitales y los recursos sociales en adultos. Sus 200 ítems se asignan a las siguientes categorías: salud física, vivienda/vecindario, finanzas, trabajo, pareja, hijos, familia extensa, y amigos y actividades de ocio. Dentro de cada categoría, se distingue entre los sucesos vitales negativos, los sucesos vitales positivos, los estresores y los recursos. Las preguntas relacionadas a los sucesos vitales son de naturaleza dicotómica y se responden con "Sí" o "No". Con relación a los estresores y recursos, existen dos opciones distintas: por un lado, una escala Likert de cuatro valores que comprenden "Definitivamente sí" hasta "Definitivamente no" y, por otro lado, una escala Likert de cinco valores que abarca "Nunca" hasta "A menudo". En el estudio de adaptación argentina, el coeficiente Alpha de Cronbach fue de .85

Ya en uno de los primeros estudios que se realizó con la versión original de dicho instrumento, Daniels y Moos (1990) mostraron que, en comparación con individuos sanos, los individuos deprimidos reportaron más estresores y menos recursos sociales. Los eventos negativos de la vida contribuyeron con una 
variación única a ambas muestras. Debido a ello, no se utilizaron en el presente estudio los ítems correspondientes a los sucesos vitales, sino solamente las escalas que hacen referencia a los estresores y recursos correspondientes al trabajo (6 ítems sobre los estresores y 6 ítems sobre los recursos), la pareja (5 ítems sobre los estresores y 6 ítems sobre los recursos), hijos (10 ítems sobre los estresores y 6 ítems sobre los recursos) y amigos (5 ítems sobre los estresores y 7 ítems sobre los recursos).

Las Work-Family Conflict and FamilyWork Conflict Scales (WFC-FWC-S) fueron desarrolladas por Netemeyer, Boles y McMurrian (1996) y evalúan dos factores: por un lado $W F C$, quiere decir el efecto que los conflictos laborales tienen sobre la vida familiar (por ejemplo, "La cantidad de tiempo que ocupa mi trabajo dificulta el cumplimiento de las responsabilidades familiares.") y, por otro lado $F W C$, quiere decir el efecto que los conflictos familiares tienen sobre el desempeño de las actividades laborales (por ejemplo, "Las cosas que quiero hacer en el trabajo no se hacen debido a las demandas de mi familia o mi cónyuge / pareja."). Cada factor dispone de cinco ítems, los cuales se responden en una escala Likert de siete puntos que abarca los valores $1=$ Totalmente en desacuerdo hasta $7=$ Totalmente de acuerdo. En el estudio de traducción y validación argentina del instrumento, se obtuvieron unas consistencias internas de $0.95 \leq \alpha \leq .96$ (Schetsche et al., en prensa).

Procedimiento: Con el fin de recolectar los datos, se recurrió a la plataforma digital de Google Forms ${ }^{\oplus}$. La página inicial del cuestionario informó sobre los datos de contacto de los directores del estudio, sobre la posibilidad de retirarse en cualquier momento de la investigación. Tras la aceptación del consentimiento informado, se accedió a los cuestionarios que serán descritos a continuación. A través de las redes sociales Facebook, Instagram y WhatsApp, se realizó el reclutamiento de los participantes.

\section{Análisis factorial exploratorio, medida de purificación $y$ análisis de consistencias internas}

Debido a que, en el presente estudio, se utilizó un total de cuatro instrumentos psicométricos, se llevó a cabo una medida de purificación para prevenir que determinados ítems pudieran cargar simultáneamente en varios factores.

Con el fin de realizar la reducción de ítems, se utilizó como criterio el índice de complejidad de Hofmann (1978). Este índice se calcula a la hora de realizar un análisis factorial exploratorio con el paquete psych de Revelle (2019) y representa el número promedio de variables latentes necesarias para explicar las variables manifiestas. De esta manera, una solución de estructura simple perfecta tiene una complejidad de valor uno, ya que cada ítem carga solamente en un único factor. Cuando un ítem carga simultáneamente en varios factores, el índice de complejidad de dicho ítem será mayor a uno.

Teniendo en cuenta que los 83 ítems iniciales incumplían con el supuesto de normalidad multivariante, se efectuó la 
secuencia de análisis factoriales exploratorios con el método de los ejes principales, siguiendo así las sugerencias de Fabrigar, Wegener, MacCallum y Strahan (1999). De acuerdo a las indicaciones de Costello y Osborne (2005), se utilizó un análisis paralelo con método de rotación Oblimin. De esta manera, se realizó la secuencia de análisis factoriales exploratorios comprendiendo el total de 14 variables. Basado en lo expresado, se eliminaba siempre aquel ítem que mayor índice de complejidad representaba $\mathrm{y}$, posteriormente, se realizaba otro análisis factorial exploratorio para poder determinar el siguiente ítem a eliminar. A través de la aplicación de dicho método, se prosiguió hasta alcanzar la cantidad de cuatro ítems por cada factor.

A continuación, se compararon las consistencias internas ( $\alpha$ y $\omega$ ) de los instrumentos originales y tras la medida de purificación. Para ello, se tuvieron en cuenta los rangos indicados por Hinton, McMurray y Brownlow (2014) y Dunn, Baguley y Brunsden (2014).

\section{Consideraciones con respecto al tamaño muestral y el trabajo con modelos de ecuaciones estructurales}

Yu (2002) encontró en modelos de $\mathrm{N}=100$ adecuados criterios de corte cuando los datos seguían una distribución con normalidad multivariante y Marsh, Hau y Wen (2004) obtuvieron resultados similares con muestras de $\mathrm{N}=150$. Por otro lado, Hoyle y Gottfredson (2015) afirman que un tamaño muestral inferior a 200 podría ser crítico, pero resaltan a su vez que existen condiciones específicas en las que incluso una muestra tan pequeña como $\mathrm{N}=$ 50 puede producir resultados confiables. Para que esto suceda, los autores dan las siguientes recomendaciones: (a) deben utilizarse todos los datos posibles, por lo que deben utilizarse determinados métodos para el tratamiento de valores faltantes; (b) deben optimizarse los datos con el fin de alcanzar una distribución con normalidad multivariante. Con este fin, recomiendan el uso de transformaciones, entre otros; (c) los indicadores más confiables de variables latentes se asocian con menos problemas de estimación y mayor poder estadístico, por lo que recomiendan utilizar tres o más ítems por cada factor para maximizar las consistencias internas; (d) deben incrementarse los grados de libertad y/o disminuirse los parámetros que deben ser estimados.

Basado en lo expresado, existe evidencia de que los modelos de ecuaciones estructurales pueden ser utilizados incluso con muestras pequeñas, siempre que los datos representen una distribución normal multivariante.

\section{Transformaciones $y \quad$ normalidad multivariante}

Siguiendo las indicaciones de Byrne (2016), los modelos de ecuaciones estructurales se basan en el cómputo de las covarianzas y éstas son sensibles al incumplimiento de la normalidad multivariante con respecto a la curtosis. Debido al tamaño muestral del presente estudio y la necesidad de trabajar con datos que siguen una distribución con normalidad multivariante (Hoyle \& 
Gottfredson, 2015; Marsh et al., 2004; Yu, 2002), se realizó otro test de Mardia (1970) con los 14 factores que se habían calculado a raíz de los 56 ítems resultantes de la medida de purificación. La curtosis multivariante de estos factores no representaba una distribución normal $(p<.05)$, por lo que se recurrió a la utilización de las transformaciones de Yeo y Johnson (2000). En este punto, debe resaltarse que, a raíz de estas transformaciones, las unidades de medida serán totalmente diferentes a los datos originales y pueden causar cierta confusión, pues cada variable tendrá $M=0$ y $D S=1$. Como resultado, la curtosis multivariante representó una distribución normal con $p=$ 0.087 .

Teniendo en cuenta lo expresado y siguiendo las sugerencias de Yu (2002), se procedió a evaluar los ajustes del modelo a través del método de máxima verosimilitud, ML. Según las indicaciones de Hu y Bentler (1999), podemos considerar un modelo como adecuado cuando su ajuste toma los siguientes valores: Error de Aproximación Cuadrático Medio (RMSEA) $\leq .06$, Residuo Medio Cuadrático Estandarizado (SRMR) $\leq$ .08, Índice de Ajuste Comparativo (CFI) $\geq$ .95 , Índice de Tucker Lewis (TLI) $\geq .95$.

\section{Establecimiento del modelo de ecuaciones estructurales}

Para poder establecer el modelo de ecuaciones estructurales, se siguieron las sugerencias de Heinze, Wallisch y Dunkler (2018), utilizando el método de Backwards Elimination. Este procedimiento comienza con el modelo global insesgado. En el segundo paso, se elimina la variable independiente con el mayor valor de probabilidad $\mathrm{p}$ y se vuelve a evaluar el modelo. De esta manera, se utilizaron (a) los síntomas como variables dependientes y recursos y estresores como variables independientes. Tras la aplicación del método de Backwards Elimination, (b) se utilizaron los recursos como variables dependientes los demás recursos y estresores como variables independientes. Se aplicó nuevamente el método descrito y, finalmente, (c) se utilizaron los estresores como variables dependientes y los demás estresores como variables independientes.

\section{Análisis de datos}

Se recurrió al test de Mardia (1970) para analizar la normalidad multivariante y, para ello, se utilizó el paquete $M V N$ de Korkmaz, Goksuluk y Zararsiz (2014). Con el fin de realizar las transformaciones de Yeo y Johnson (2000), se utilizó el paquete bestNormalize de Peterson y Cavanaugh (2020). El análisis factorial exploratorio, el cálculo de las consistencias internas ( $\alpha$ y $\omega$ ) y de los índices de complejidad de Hofmann (1978) se realizaron con la ayuda del paquete psych de Revelle (2019). A través del paquete lavaan de Rosseel (2012), se desarrolló el modelo de ecuaciones estructurales (SEM) para realizar un análisis de sendero (Kline, 2015; Martín \& Fernández, 1978; Raykov \& Marcoulides, 2006) y el posterior análisis de invarianza factorial. Todos los paquetes mencionados forman parte del software $\mathrm{R}$ de Core Team (2020) y, para todos los cálculos indicados, se utilizó el valor de probabilidad $p \leq .05$.

\section{Resultados}


Análisis factorial exploratorio, medida de purificación $y$ análisis de las consistencias internas

Tras la realización de los análisis factoriales exploratorios y la aplicación de la medida de purificación, la media de todos los índices de complejidad pudo ser mejorada, pues disminuyó desde 2.032 hasta el valor final de 1.602. Aunque existía la posibilidad de continuar la medida de purificación y reducir los instrumentos hasta un total de tres ítems por cada dimensión, no se llevó a cabo esa operación, ya que hubiera perjudicado las consistencias internas de los factores.

Tras la eliminación, la asignación de ítems del SA-45 quedó como sigue: depresión ítems 1, 2, 4, 5 y ansiedad ítems 6, 7, 8, 10. La asignación de ítems del SISCO quedó de la siguiente forma: estresores ítems 1, 2, 4, 5 y estrategias ítems 11, 12,13, 14. Con respecto al LISRES, estos ítems representan las ocho dimensiones que fueron utilizadas en el presente estudio: estresores del trabajo ítems 1, 2, 3, 4; recursos trabajo ítems 1, 2, 3, 4; estresores pareja ítems $2,3,4,5$; recursos pareja ítems 1, 2, 4, 5; estresores hijos ítems 2, 3, 5, 8; recursos hijos ítems 2, 3, 5, 6; estresores amigos ítems 1, 2, 3, 4; recurso amigos ítems 4, 5, 6, 9. El Work-Family Conflict and Family-Work Conflict Scales quedó con la siguiente asignación de ítems: FWC ítems 1 , 2, 3, 4 y WFC ítems 2, 3, 4, 5. La Tabla 1 representa las consistencias de los instrumentos originales y tras la medida de purificación. Como podemos observar, todos los valores se encontraron en unos rangos adecuados (Dunn et al., 2014; Hinton et al., 2014)

Tabla 1

Consistencias internas de los instrumentos originales y tras la medida de purificación

\begin{tabular}{llcccc}
\hline Instrumento & $\begin{array}{l}\text { Nombre } \\
\text { dimensión }\end{array}$ & $\begin{array}{l}\boldsymbol{\alpha} \\
\text { instrumen } \\
\text { to original }\end{array}$ & $\begin{array}{l}\boldsymbol{\alpha} \text { tras la } \\
\text { medida de } \\
\text { purificación }\end{array}$ & $\begin{array}{l}\boldsymbol{\omega} \\
\text { instrumen } \\
\text { to original }\end{array}$ & $\begin{array}{l}\text { w tras la } \\
\text { medida de } \\
\text { purificación }\end{array}$ \\
\hline SA-34 & & & & & \\
& Depresión & 0,803 & 0,757 & 0,865 & 0,811 \\
SISCO & Ansiedad & 0,840 & 0,854 & 0,895 & 0,891 \\
& Estresor & & & & \\
& pandemia & 0,813 & 0,796 & 0,854 & 0,826 \\
& Estrategia & & & & \\
pandemia & 0,665 & 0,682 & 0,757 & 0,771 \\
& & & & & \\
& Estresor trabajo & 0,779 & 0,767 & 0,878 & 0,839 \\
& Recurso trabajo & 0,781 & 0,802 & 0,882 & 0,849 \\
& Estresor pareja & 0,823 & 0,876 & 0,877 & 0,903 \\
& Recurso pareja & 0,844 & 0,788 & 0,892 & 0,851 \\
& Estresor hijos & 0,916 & 0,902 & 0,941 & 0,915 \\
& Recurso hijos & 0,819 & 0,793 & 0,892 & 0,830 \\
& Estresor amigos & 0,717 & 0,709 & 0,799 & 0,775 \\
& Recurso amigos & 0,823 & 0,868 & 0,871 & 0,888
\end{tabular}


WFC-FWC-S

\section{Conflicto \\ familia-trabajo \\ Conflicto \\ trabajo-familia}

Media de todas las dimensiones
Establecimiento del modelo de ecuaciones estructurales

\section{Síntomas como variables dependientes y recursos y estresores como variables independientes}

En primer lugar, se utilizaron los síntomas como variables dependientes y los recursos y estresores como variables independientes. Como puede observar en la Tabla 2, solamente recursos pareja $y$ estresores pandemia tuvieron efectos directos sobre la depresión. Por otro lado, recursos pareja, estresores hijos y estresores pandemia tuvieron efectos directos sobre la ansiedad.

\section{Tabla 2}

Resultados de regresiones utilizando síntomas como criterios y recursos y estresores como predictores

\begin{tabular}{|c|c|c|c|c|c|c|c|c|}
\hline \multicolumn{9}{|c|}{$95 \%$ IC } \\
\hline $\begin{array}{l}\text { Criterio } \\
\text { Depresión }\end{array}$ & Predictores & $b$ & $L I$ & $L S$ & $S E B$ & $\mathrm{z}$ & $\operatorname{Beta}(\beta)$ & $\mathrm{p}$ \\
\hline & Recursos pareja & $-0,277$ & $-0,435$ & $-0,119$ & 0,081 & $-3,436$ & $-0,277$ & $0,001^{* *}$ \\
\hline & $\begin{array}{l}\text { Estresores } \\
\text { pandemia }\end{array}$ & 0,159 & 0,027 & 0,291 & 0,067 & 2,367 & 0,159 & $0,018^{*}$ \\
\hline \multicolumn{9}{|l|}{ Ansiedad } \\
\hline & Recursos pareja & $-0,271$ & $-0,435$ & $-0,107$ & 0,084 & $-3,235$ & $-0,271$ & $0,001^{* *}$ \\
\hline & Estresores hijos & 0,221 & 0,065 & 0,377 & 0,080 & 2,775 & 0,221 & $0,006^{* *}$ \\
\hline & $\begin{array}{l}\text { Estresores } \\
\text { pandemia }\end{array}$ & 0,196 & 0,059 & 0,332 & 0,070 & 2,804 & 0,196 & $0,005^{* *}$ \\
\hline
\end{tabular}

Notas. $\mathrm{n}=182{ }^{* *} \mathrm{p}<.01 ;^{*} \mathrm{p}<.05$; IC, Intervalo de confianza; LI, límite inferior; LS, límite superior.

Recursos como variables dependientes y los demás recursos y estresores como variables independientes

Al analizar el contenido de la Tabla 3, pueden encontrarse numerosas asociaciones inesperadas. Con respecto a los recursos del trabajo como variable dependiente, es llamativo que los estresores del trabajo tengan un efecto positivo, quiere decir que la aumentada percepción de estresores laborales aumenta también la percepción de recursos laborales. 
Tabla 3

Resultados de regresiones utilizando recursos como criterios y los demás recursos y estresores como predictores

\begin{tabular}{|c|c|c|c|c|c|c|c|c|}
\hline \multirow[b]{2}{*}{ Criterio } & \multirow[b]{2}{*}{ Predictores } & \multirow[b]{2}{*}{$b$} & \multicolumn{2}{|c|}{$95 \%$ IC } & \multirow[b]{2}{*}{$S E B$} & \multirow[b]{2}{*}{$\mathrm{z}$} & \multirow[b]{2}{*}{$\operatorname{Beta}(\beta)$} & \multirow[b]{2}{*}{$\mathrm{p}$} \\
\hline & & & $L I$ & $L S$ & & & & \\
\hline \multicolumn{9}{|c|}{ Recursos trabajo } \\
\hline & Estresores trabajo & 0,343 & 0,201 & 0,485 & 0,072 & 4,743 & 0,343 & $0,000^{* *}$ \\
\hline & Recursos amigos & 0,196 & 0,046 & 0,346 & 0,077 & 2,564 & 0,196 & $0,010^{*}$ \\
\hline \multicolumn{9}{|c|}{ Recursos pareja } \\
\hline & Estresores pareja & $-0,464$ & $-0,597$ & $-0,330$ & 0,068 & $-6,805$ & $-0,464$ & $0,000^{* *}$ \\
\hline & Estresores amigos & 0,181 & 0,053 & 0,308 & 0,065 & 2,782 & 0,181 & $0,005^{* *}$ \\
\hline & Recursos amigos & 0,214 & 0,084 & 0,345 & 0,067 & 3,212 & 0,214 & $0,001^{* *}$ \\
\hline \multicolumn{9}{|c|}{ Recursos hijos } \\
\hline & Recursos pareja & 0,184 & 0,027 & 0,342 & 0,080 & 2,298 & 0,184 & $0,022^{*}$ \\
\hline & Estresores hijos & $-0,253$ & $-0,400$ & $-0,106$ & 0,075 & $-3,374$ & $-0,253$ & $0,001^{* *}$ \\
\hline & Estrategias pandemia & 0,190 & 0,055 & 0,326 & 0,069 & 2,751 & 0,190 & $0,006^{* *}$ \\
\hline \multicolumn{9}{|c|}{ Recursos amigos } \\
\hline & Recursos trabajo & 0,178 & 0,042 & 0,313 & 0,069 & 2,564 & 0,178 & $0,010^{*}$ \\
\hline & Recursos pareja & 0,250 & 0,097 & 0,403 & 0,078 & 3,212 & 0,250 & $0,001^{* *}$ \\
\hline & Estresores amigos & $-0,183$ & $-0,321$ & $-0,046$ & 0,070 & $-2,611$ & $-0,183$ & $0,009^{* *}$ \\
\hline
\end{tabular}

Notas. $\mathrm{n}=182{ }^{* *} \mathrm{p}<.01 ;^{*} \mathrm{p}<.05$; IC, Intervalo de confianza; LI, límite inferior; LS, límite superior.

Estresores como variables dependientes y los demás estresores como variables independientes

Como último paso previo al establecimiento del primer modelo estructural, se calcularon los efectos que los recursos tuvieron entre ellas. La Tabla 4 representa los resultados correspondientes, de los que se resalta, con el único signo negativo, la relación recíproca entre los estresores amigos y los conflictos familia-trabajo.

Tabla 4

Resultados de regresiones utilizando estresores como criterios y los demás estresores como predictores

\begin{tabular}{|c|c|c|c|c|c|c|c|c|}
\hline \multirow[b]{2}{*}{ Criterio } & \multirow[b]{2}{*}{ Predictores } & \multirow[b]{2}{*}{$\boldsymbol{b}$} & \multicolumn{2}{|c|}{$95 \%$ IC } & \multirow[b]{2}{*}{$S E B$} & \multirow[b]{2}{*}{$\mathbf{z}$} & \multirow[b]{2}{*}{$\begin{array}{c}\text { Beta } \\
(\boldsymbol{\beta})\end{array}$} & \multirow[b]{2}{*}{$\mathbf{p}$} \\
\hline & & & $L I$ & $L S$ & & & & \\
\hline \multicolumn{9}{|c|}{ Estresores trabajo } \\
\hline & Conflicto trabajo-familia & 0,412 & 0,253 & 0,571 & 0,081 & 5,086 & 0,412 & $0,000^{* *}$ \\
\hline \multicolumn{9}{|c|}{ Estresores pareja } \\
\hline & Estresores hijos & 0,193 & 0,047 & 0,340 & 0,075 & 2,582 & 0,193 & $0,010^{*}$ \\
\hline & Estresores amigos & 0,299 & 0,164 & 0,435 & 0,069 & 4,332 & 0,299 & $0,000^{* *}$ \\
\hline
\end{tabular}


Estresores hijos

$\begin{array}{llllllll}\text { Estresores pareja } & 0,183 & 0,044 & 0,321 & 0,071 & 2,582 & 0,183 & 0,010^{*} \\ \text { Conflicto familia-trabajo } & 0,274 & 0,125 & 0,424 & 0,076 & 3,592 & 0,274 & 0,000^{* *} \\ \text { Estrategias pandemia } & 0,150 & 0,018 & 0,281 & 0,067 & 2,230 & 0,150 & 0,026^{*}\end{array}$

Estresores amigos

$\begin{array}{lrrrrrrr}\text { Estresores pareja } & 0,312 & 0,171 & 0,454 & 0,072 & 4,332 & 0,312 & 0,000^{* *} \\ \text { Conflicto trabajo-familia } & -0,236 & -0,400 & -0,072 & 0,084 & -2,825 & -0,236 & 0,005^{* *}\end{array}$

Conflicto trabajo-familia

$\begin{array}{lrrrrrrr}\text { Estresores trabajo } & 0,302 & 0,186 & 0,418 & 0,059 & 5,086 & 0,302 & 0,000^{* *} \\ \text { Estresores amigos } & -0,178 & -0,301 & -0,054 & 0,063 & -2,825 & -0,178 & 0,005^{* *} \\ \text { Conflicto familia-trabajo } & 0,424 & 0,297 & 0,552 & 0,065 & 6,545 & 0,424 & 0,000^{* *}\end{array}$

Conflicto familia-trabajo

Estresores hijos

$$
0,241
$$

0,110

0,373

$0,067 \quad 3,592$

0,241

$0,000^{* *}$

Conflicto trabajo-familia

0,449

0,314

$0,583 \quad 0,069$

6,545

$0,449 \quad 0,000^{* *}$

Estresores pandemia

Estrategias pandemia

$$
0,211 \quad 0,068
$$

0,354

0,073

2,892

$0,2110,004^{* *}$

Estrategias pandemia

Estresores hijos

$0,178 \quad 0,022$

0,334

0,080

2,230

0,178

$0,026^{*}$

Estresores pandemia

0,208

0,067

$0,349 \quad 0,072$

2,892

$0,208 \quad 0,004^{* *}$

Notas. $\mathrm{n}=182 ;{ }^{* *} \mathrm{p}<.01 ;{ }^{*} \mathrm{p}<.05$; IC, Intervalo de confianza; LI, límite inferior; LS, límite superior.

\section{Establecimiento del primer modelo estructural y trabajo sobre los índices de modificación}

Al analizar la Tabla 3 y Tabla 4, se han encontrado numerosos efectos recíprocos: recurso trabajo con recursos amigos, recursos pareja con recursos amigos, estresores trabajo con conflicto trabajofamilia, estresores pareja con estresores hijos, estresores pareja con estresores amigos, estresores hijos con conflicto familia-trabajo, estresores hijos con estrategias pandemia, estresores amigos con conflicto trabajo-familia, conflicto trabajofamilia con conflicto familia-trabajo y estresores pandemia con estrategias pandemia. Aunque pueden existir varios caminos recíprocos legítimos, Byrne (2016) afirma que no todos los caminos pueden ser de interés sustancial en un estudio, por lo que sugiere el uso de un flujo de interés. Este se representa en la Ilustración 2 y es de izquierda a derecha, de acuerdo con los objetivos del presente estudio. De esta manera, pueden encontrarse los estresores en el margen izquierdo de la ilustración, en el medio los recursos y, en el margen derecho, los síntomas.

A raíz de los efectos directos que fueron descritos, se estableció el primer modelo estructural que se representa en la Ilustración 2. En este punto, debe hacerse énfasis en el método que se ha utilizado para ello. En el caso de los efectos directos entre dos recursos o entre dos estresores, se continuó trabajando, como primer criterio, con el efecto directo con menor valor de probabilidad $p$ y, si ambos efectos tenían el mismo valor $p$, se prosiguió con el efecto con mayor valor estandarizado $(\beta)$. 


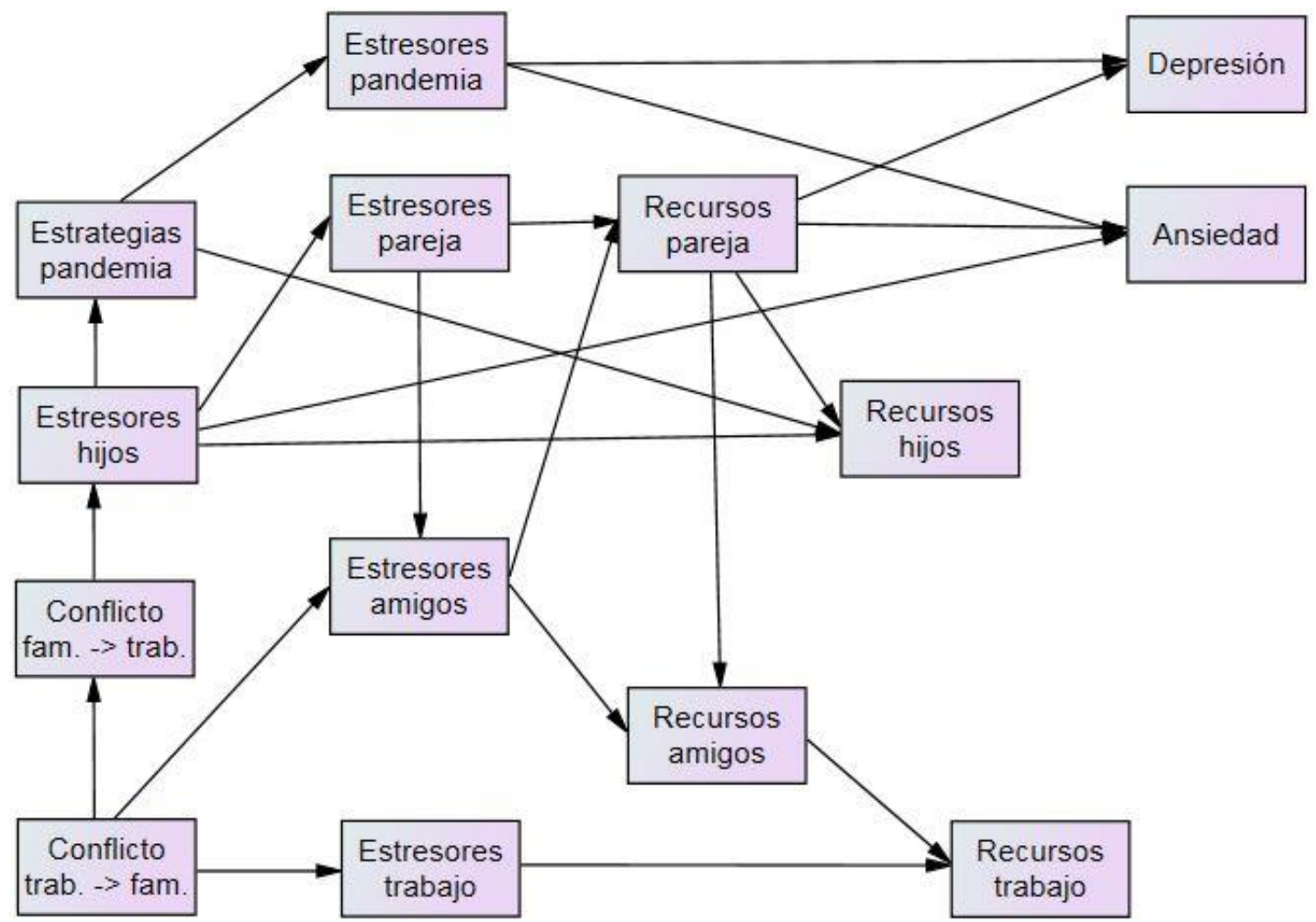

Figura 2. Primer modelo estructural a raíz de los efectos directos descritos en la Tabla 3 y Tabla 4.

A partir de este momento, se continuó trabajando con los índices de modificación utilizando el método propuesto por Saris, Satorra y Van Der Veld (2009). Como primera modificación, se agregó un efecto directo de los conflictos familia-trabajo sobre la depresión. La segunda modificación comprendió el efecto directo de los estresores hijos sobre recursos trabajo. El efecto directo de los amigos como estresores sobre la depresión consistió en la tercera y última modificación.

La Tabla 5 representa los índices de ajuste del modelo inicial y de los tres modelos tras sus respectivas modificaciones. Utilizando los valores de corte propuestos por $\mathrm{Hu}$ y Bentler (1999), podemos afirmar que se ha desarrollado un modelo significativamente exacto.

\section{Tabla 5}

Índices de ajuste del modelo estructural

\begin{tabular}{lcccccccc}
\hline Modelos competidores & $\chi^{2}$ ML & df & $\boldsymbol{\chi 2} / \mathbf{d f}$ & $\mathbf{p}$ & RMSEA (90\% CI) & SRMR & CFI & TLI \\
\hline Modelo inicial & 81,698 & 63 & 1,297 & 0,057 & $0,040(0,000-0,063)$ & 0,074 & 0,957 & 0,938 \\
Modelo tras la modificación no-1 & 72,033 & 62 & 1,162 & 0,180 & $0,030(0,000-0,056)$ & 0,067 & 0,977 & 0,966 \\
Modelo tras la modificación no2 & 65,902 & 61 & 1,080 & 0,311 & $0,021(0,000-0,051)$ & 0,062 & 0,989 & 0,983 \\
Modelo final & 60,176 & 60 & 1,003 & 0,469 & $0,004(0,000-0,045)$ & 0,060 & 1,000 & 0,999 \\
\hline
\end{tabular}

Notas. $\mathrm{n}=182 ; \chi 2 \mathrm{ML}$ - Chi-cuadrado utilizando el método de máxima verosimilitud; df, grados de libertad; RMSEA, error cuadrático medio de aproximación; SRMR, residuo estandarizado cuadrático medio, CFI, índice de ajuste comparativo; TLI, Tucker Lewis Index.. 
La Ilustración 3 representa el modelo final, el cual explica el 19,9\% de la varianza de depresión y el $12,9 \%$ de la varianza de ansiedad. Con respecto a las demás variables, destacaron la pareja como recurso con un $27,0 \%$ de varianza explicada, los conflictos familia-trabajo con un $23,3 \%$ de varianza explicada, los amigos como recursos con un 19,3\% de varianza explicada, los hijos como recursos con un $17,9 \%$ de varianza explicada. Al analizar el contenido de la Ilustración 3, varios elementos llaman la atención: conflicto trabajo-familia es el único factor que no recibe ningún efecto por parte de las demás variables. Estresores hijos es la variable con mayor número de efectos directos $(\mathrm{n}=5)$ : sobre estrategias pandemia, estresores pareja, ansiedad, recursos hijos y recursos trabajo. Así mismo, la depresión es el factor con el mayor número de efectos recibidos $(\mathrm{n}=4)$ : de estresores pandemia, recursos pareja, conflicto familia-trabajo y estresores amigos.

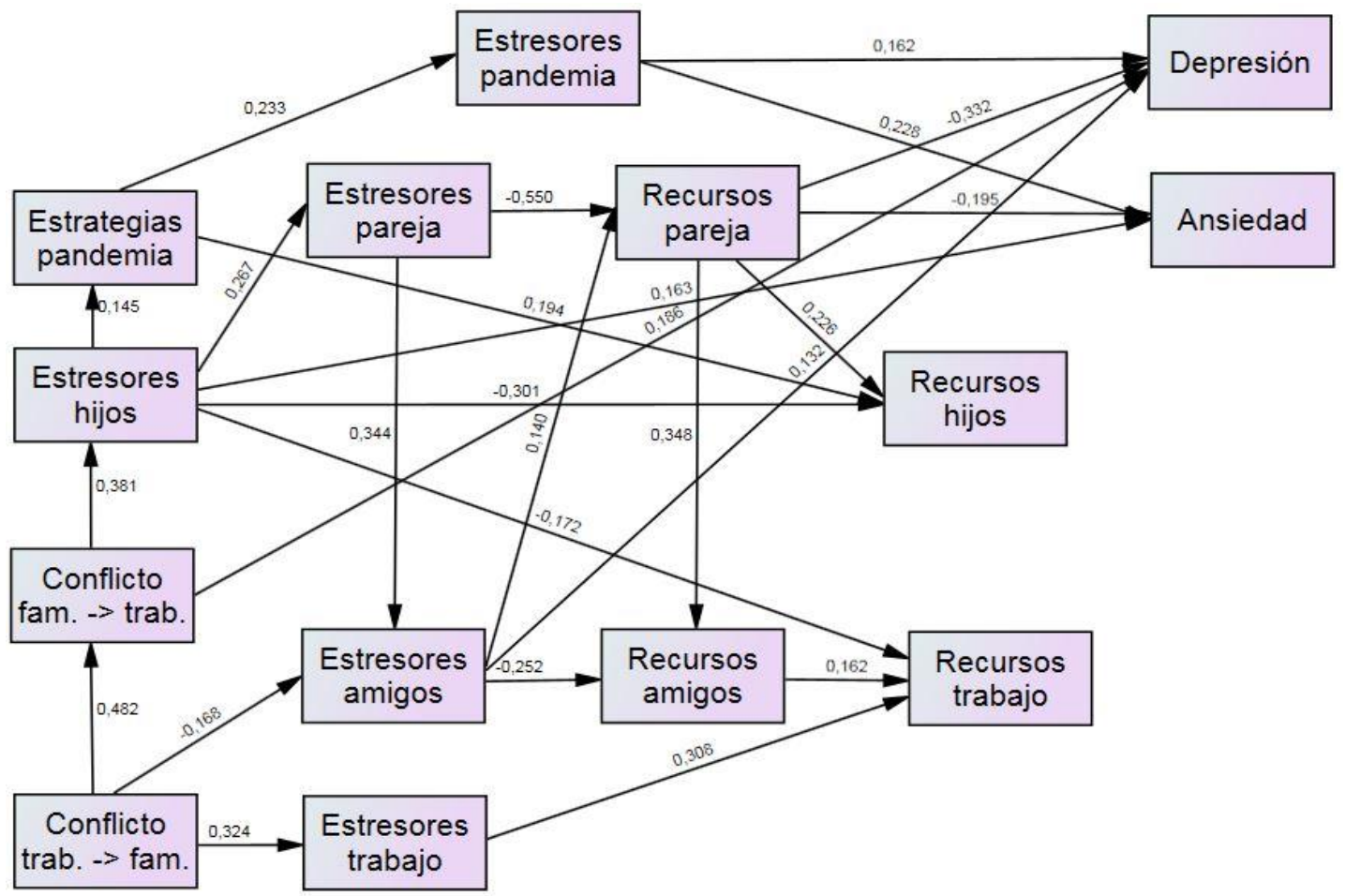

Figura 3. Modelo estructural final tras las seis modificaciones

La Tabla 6 muestra todos los valores correspondientes a las regresiones del modelo final. De las asociaciones con mayores valores estandarizados $(\beta)$, destacaron los siguientes efectos directos: estresores pareja sobre recursos pareja, conflictos trabajo-familia sobre conflictos familia-trabajo, conflicto familia-trabajo sobre estresores hijos, recursos pareja sobre recursos amigos, estresores pareja sobre estresores amigos, recursos pareja sobre depresión.

$\mathrm{Al}$ analizar las variables relacionadas con la pandemia del COVID-19, es llamativo que la utilización de estrategias para evitar el contagio aumenta la percepción de estresores de la pandemia. 
Tabla 6

Resultados de regresiones del modelo final

\begin{tabular}{|c|c|c|c|c|c|c|c|c|}
\hline \multirow[b]{2}{*}{ Criterio } & \multirow[b]{2}{*}{ Predictores } & \multirow[b]{2}{*}{$\boldsymbol{b}$} & \multicolumn{2}{|c|}{$95 \%$ IC } & \multirow[b]{2}{*}{$S E B$} & \multirow[b]{2}{*}{$\mathbf{Z}$} & \multirow[b]{2}{*}{ Beta $(\beta)$} & \multirow[b]{2}{*}{ p } \\
\hline & & & $L I$ & $L S$ & & & & \\
\hline \multicolumn{9}{|c|}{ Depresión } \\
\hline & Recursos pareja & $-0,328$ & $-0,456$ & $-0,200$ & 0,065 & $-5,007$ & $-0,332$ & $0,000^{* *}$ \\
\hline & Estresores pandemia & 0,160 & 0,032 & 0,288 & 0,065 & 2,452 & 0,162 & $0,014^{*}$ \\
\hline & Conflicto familia-trabajo & 0,184 & 0,076 & 0,292 & 0,055 & 3,335 & 0,186 & $0,001^{* *}$ \\
\hline & Estresores amigos & 0,130 & 0,025 & 0,235 & 0,054 & 2,425 & 0,132 & $0,015^{*}$ \\
\hline \multicolumn{9}{|c|}{ Ansiedad } \\
\hline & Recursos pareja & $-0,193$ & $-0,329$ & $-0,058$ & 0,069 & $-2,802$ & $-0,195$ & $0,005^{* *}$ \\
\hline & Estresores pandemia & 0,225 & 0,092 & 0,359 & 0,068 & 3,311 & 0,228 & $0,001^{* *}$ \\
\hline & Estresores hijos & 0,161 & 0,046 & 0,277 & 0,059 & 2,744 & 0,163 & $0,006^{* *}$ \\
\hline \multicolumn{9}{|c|}{ Recursos hijos } \\
\hline & Recursos pareja & 0,224 & 0,093 & 0,355 & 0,067 & 3,342 & 0,226 & $0,001^{* *}$ \\
\hline & Estrategias pandemia & 0,192 & 0,063 & 0,321 & 0,066 & 2,911 & 0,194 & $0,004^{* *}$ \\
\hline & Estresores hijos & $-0,297$ & $-0,430$ & $-0,165$ & 0,068 & $-4,398$ & $-0,301$ & $0,000^{* *}$ \\
\hline \multicolumn{9}{|c|}{ Recursos trabajo } \\
\hline & Recursos amigos & 0,161 & 0,028 & 0,295 & 0,068 & 2,367 & 0,162 & $0,018^{*}$ \\
\hline & Estresores trabajo & 0,307 & 0,173 & 0,440 & 0,068 & 4,504 & 0,308 & $0,000^{* *}$ \\
\hline & Estresores hijos & $-0,172$ & $-0,306$ & $-0,038$ & 0,068 & $-2,517$ & $-0,172$ & $0,012^{*}$ \\
\hline \multicolumn{9}{|c|}{ Recursos pareja } \\
\hline & Estresores pareja & $-0,549$ & $-0,681$ & $-0,418$ & 0,067 & $-8,181$ & $-0,550$ & $0,000^{* *}$ \\
\hline & Estresores amigos & 0,139 & 0,008 & 0,270 & 0,067 & 2,081 & 0,140 & $0,037^{*}$ \\
\hline \multicolumn{9}{|c|}{ Recursos amigos } \\
\hline & Recursos pareja & 0,348 & 0,217 & 0,479 & 0,067 & 5,218 & 0,348 & $0,000^{* *}$ \\
\hline & Estresores amigos & $-0,252$ & $-0,382$ & $-0,121$ & 0,066 & $-3,786$ & $-0,252$ & $0,000^{* *}$ \\
\hline \multicolumn{9}{|c|}{ Estresores pandemia } \\
\hline & Estrategias pandemia & 0,233 & 0,092 & 0,374 & 0,072 & 3,235 & 0,233 & $0,001^{* *}$ \\
\hline \multicolumn{9}{|c|}{ Estresores pareja } \\
\hline & Estresores hijos & 0,267 & 0,127 & 0,407 & 0,071 & 3,743 & 0,267 & $0,000^{* *}$ \\
\hline \multicolumn{9}{|c|}{ Estresores amigos } \\
\hline & Estresores pareja & 0,345 & 0,209 & 0,480 & 0,069 & 4,993 & 0,344 & $0,000^{* *}$ \\
\hline & Conflicto trabajo-familia & $-0,168$ & $-0,304$ & $-0,033$ & 0,069 & $-2,437$ & $-0,168$ & $0,015^{*}$ \\
\hline \multicolumn{9}{|c|}{ Estresores trabajo } \\
\hline & Conflicto trabajo-familia & 0,324 & 0,186 & 0,461 & 0,070 & 4,618 & 0,324 & $0,000^{* *}$ \\
\hline \multicolumn{9}{|c|}{ Estrategias pandemia } \\
\hline & Estresores hijos & 0,145 & 0,001 & 0,289 & 0,073 & 1,976 & 0,145 & $0,048^{*}$ \\
\hline \multicolumn{9}{|c|}{ Estresores hijos } \\
\hline & Conflicto familia-trabajo & 0,381 & 0,247 & 0,515 & 0,069 & 5,558 & 0,381 & $0,000^{* *}$ \\
\hline Conflicts & familia-trabajo & & & & & & & \\
\hline & Conflicto trabajo-familia & 0,482 & 0,355 & 0,610 & 0,065 & 7,429 & 0,482 & $0,000 * *$ \\
\hline
\end{tabular}

Notas. $\mathrm{n}=182 ;{ }^{* *} \mathrm{p}<.01{ }^{*} \mathrm{p}<.05$; IC, Intervalo de confianza; LI, límite inferior; LS, límite superior.

Con el fin de profundizar en el análisis de las asociaciones, se calculó la matriz de efectos totales cuyos resultados pueden observarse en la Tabla 7. La pareja como recurso tiene los efectos totales más significativos para reducir los síntomas. Por otro lado, los estresores pandemia tienen considerables efectos sobre la depresión y ansiedad, la pareja como estresor sobre la depresión, los conflictos familia-trabajo sobre depresión, y los hijos como estresores sobre ansiedad. Así mismo, se advierte que 
el conflicto trabajo-familia tiene las asociaciones más considerables con los demás estresores. Con relación a los efectos totales más relevantes del modelo en su conjunto, debe destacarse la pareja como estresor con un efecto de signo negativo sobre la pareja como recurso, los conflictos trabajo-familia con un efecto de signo

Tabla 7

Matriz de efectos totales estandarizados positivo sobre conflictos familia-trabajo, la pareja como recurso con un efecto de signo positivo sobre los amigos como recurso, la pareja como estresores con un efecto de signo positivo sobre los amigos como estresores y el conflicto trabajo-familia sobre estresores del trabajo.

\begin{tabular}{lrrrrrrrrrc}
\hline & \multicolumn{10}{c}{ Predictores } \\
\cline { 2 - 11 } Criterios & \multirow{2}{*}{ WFC } & \multirow{2}{*}{ FWC } & $\begin{array}{r}\text { EST } \\
\text { HIJ }\end{array}$ & $\begin{array}{r}\text { EST } \\
\text { PAR }\end{array}$ & $\begin{array}{r}\text { EST } \\
\text { AMI }\end{array}$ & ESTRAT & $\begin{array}{r}\text { REC } \\
\text { PAR }\end{array}$ & $\begin{array}{c}\text { REC } \\
\text { AMI }\end{array}$ & $\begin{array}{c}\text { EST } \\
\text { TRAB }\end{array}$ & $\begin{array}{c}\text { EST } \\
\text { PAN }\end{array}$ \\
\hline (1) Conflicto familia-trabajo & 0,482 & 0,000 & 0,000 & 0,000 & 0,000 & 0,000 & 0,000 & 0,000 & 0,000 & 0,000 \\
(2) Estresores hijos & 0,184 & 0,381 & 0,000 & 0,000 & 0,000 & 0,000 & 0,000 & 0,000 & 0,000 & 0,000 \\
(3) Estresores pareja & 0,049 & 0,102 & 0,267 & 0,000 & 0,000 & 0,000 & 0,000 & 0,000 & 0,000 & 0,000 \\
(4) Estresores amigos & $-0,151$ & 0,035 & 0,092 & 0,344 & 0,000 & 0,000 & 0,000 & 0,000 & 0,000 & 0,000 \\
(5) Estrategias pandemia & 0,027 & 0,055 & 0,145 & 0,000 & 0,000 & 0,000 & 0,000 & 0,000 & 0,000 & 0,000 \\
(6) Estresores trabajo & 0,324 & 0,000 & 0,000 & 0,000 & 0,000 & 0,000 & 0,000 & 0,000 & 0,000 & 0,000 \\
(7) Estresores pandemia & 0,006 & 0,013 & 0,034 & 0,000 & 0,000 & 0,233 & 0,000 & 0,000 & 0,000 & 0,000 \\
(8) Recursos pareja & $-0,048$ & $-0,051$ & $-0,134$ & $-0,502$ & 0,140 & 0,000 & 0,000 & 0,000 & 0,000 & 0,000 \\
(9) Recursos amigos & 0,021 & $-0,027$ & $-0,070$ & $-0,261$ & $-0,204$ & 0,000 & 0,348 & 0,000 & 0,000 & 0,000 \\
(10) Recursos trabajo & 0,071 & $-0,070$ & $-0,184$ & $-0,042$ & $-0,033$ & 0,000 & 0,056 & 0,162 & 0,308 & 0,000 \\
(11) Recursos hijos & $-0,061$ & $-0,115$ & $-0,303$ & $-0,114$ & 0,032 & 0,194 & 0,226 & 0,000 & 0,000 & 0,000 \\
(12) Depresión & 0,087 & 0,210 & 0,062 & 0,212 & 0,086 & 0,038 & $-0,332$ & 0,000 & 0,000 & 0,162 \\
(13) Ansiedad & 0,041 & 0,075 & 0,197 & 0,098 & $-0,027$ & 0,053 & $-0,195$ & 0,000 & 0,000 & 0,228 \\
Media de estresores (1-7) & 0,132 & 0,084 & 0,077 & 0,049 & 0,000 & 0,033 & 0,000 & 0,000 & 0,000 & 0,000 \\
Media de recursos (8-11) & $-0,004$ & $-0,066$ & $-0,173$ & $-0,230$ & $-0,016$ & 0,049 & 0,158 & 0,041 & 0,077 & 0,000 \\
Media de síntomas (12-13) & 0,064 & 0,143 & 0,130 & 0,155 & 0,030 & 0,046 & $-0,264$ & 0,000 & 0,000 & 0,195 \\
\hline
\end{tabular}

Notas. $\mathrm{n}=182$; WFC, conflictos trabajo-familia; FWC, conflictos familia-trabajo; EST HIJ, estresores hijos; EST PAR, estresores pareja; EST AMI, estresores amigos; ESTRAT, estrategias pandemia; REC PAR, recursos pareja; REC AMI, recursos amigos; EST TRA, estresores trabajo; EST PAN, estresores pandemia.

\section{Invarianza factorial}

Se realizó un análisis multigrupo por género. En el M2, se igualaron las cargas factoriales entre grupos, en el M3 las cargas factoriales y los interceptos y, en el M4, las cargas factoriales, los interceptos y los residuos. Debido a que, en el presente estudio, no se utilizaron variables latentes, el M1 y el M2 representan los mismos valores. Así mismo, debe resaltarse que el participante que se identificó como otro no pudo ser incluido en el análisis, ya que su número no era suficiente para el método estadístico. Como puede observarse en la Tabla 8, los cambios de los índices se encontraron dentro de unos rangos adecuados con $\mid \Delta$ RMSEA $\mid \leq .015$ según Chen (2007). Basado en lo expresado anteriormente, podemos afirmar que el modelo representa invarianza factorial con relación a los géneros, quiere decir que, independientemente del género del participante, evalúa el mismo constructo. 
Tabla 8

Ajuste del modelo final y comparación de modelos con respecto al género

\begin{tabular}{|c|c|c|c|c|c|c|c|c|}
\hline \multirow[t]{2}{*}{ Modelo } & \multicolumn{4}{|l|}{$\begin{array}{l}\text { Ajuste de } \\
\text { modelo }\end{array}$} & \multicolumn{4}{|c|}{$\begin{array}{l}\text { Comparación de } \\
\text { modelos }\end{array}$} \\
\hline & $\chi 2 \mathrm{ML}$ & $\mathrm{df}$ & $\chi 2 / \mathrm{df}$ & RMSEA & $\Delta \chi 2 \mathrm{ML}$ & $\Delta \mathrm{df}$ & $\mathrm{p}$ & $\triangle \mathrm{RMSEA}$ \\
\hline $\begin{array}{l}\text { M1: } \\
\text { Configural }\end{array}$ & 173,592 & 120 & 1,447 & 0,070 & & & & \\
\hline M2: Débil & 173,594 & 120 & 1,447 & 0,070 & 0,002 & 0 & - & 0,000 \\
\hline $\begin{array}{l}\text { M3: } \\
\text { Fuerte }\end{array}$ & 220,208 & 133 & 1,656 & 0,085 & 46,614 & 13 & 0,000 & 0,015 \\
\hline $\begin{array}{l}\text { M4: } \\
\text { Estricto }\end{array}$ & 239,014 & 146 & 1,637 & 0,084 & 18,806 & 13 & 0,129 & $-0,001$ \\
\hline
\end{tabular}

Notas. $\mathrm{n}=181 ;$ femenino $=144 ;$ masculino $=37 ; \chi 2 \mathrm{ML}-$ Chi-cuadrado utilizando el método de máxima verosimilitud; df, Grados de libertad; RMSEA, error cuadrático medio de aproximación; CFI, índice de ajuste comparativo.

\section{Discusión}

Con el objetivo de analizar, por un lado, si existen estresores que fomentan mayores niveles de la depresión y la ansiedad $\mathrm{y}$, por otro lado, si existen recursos que pueden reducir estos síntomas, se desarrolló un modelo de ecuaciones estructurales significativamente exacto que describe las asociaciones entre un total de catorce variables: la pareja como recurso y como estresor, los hijos como recursos y como estresores, los amigos como recursos y estresores, los recursos y estresores del trabajo, los conflictos trabajo-familia, los conflictos familia-trabajo, y las estrategias y estresores relacionados con la pandemia del COVID-19

En primer lugar, se resalta que los estresores de la pandemia tienen el más significativo efecto sobre el total de los síntomas. Al analizar el camino de efectos que conduce hacia estos estresores, es llamativo que los hijos como estresores están asociados positivamente con el mayor uso de estrategias para evitar el contagio y, como consecuencia, tienen también una correlación positiva con la percepción de estresores de pandemia. Spinelli, Lionetti, Setti y Fasolo (2020) afirman que lidiar con el encierro es una experiencia estresante para los padres que tienen que equilibrar la vida personal, el trabajo y la educación de sus hijos. Así mismo, agregan que esta situación afecta potencialmente su capacidad para ser cuidadores de apoyo y, en consecuencia, es perjudicial para el bienestar de los niños. Además, Liu y Doan (2020) resaltan que, durante los tiempos del COVID-19, han aumentado las dos formas en las que se contagia el estrés en sí: por un lado, exponen que el estresor de un determinado entorno influye más en la habilidad de responder a estresores de otro entorno y, por otro lado, que el aumentado nivel de estrés de un miembro familiar puede aumentar más el nivel de estrés de otro familiar (Bolger, DeLongis, Kessler, \& Wethington, 1989). Teniendo en cuenta eso, la asociación entre los hijos como estresores y el mayor uso de estrategias para evitar el contagio puede entenderse como 
un ejemplo más de estos dos tipos de contagio del estrés.

Como recurso más importante para reducir los síntomas, destaca la pareja. Aun así, debe tenerse en cuenta que la pareja como estresor consiste también en uno de los factores con las asociaciones negativas más considerables con los recursos $\mathrm{y}$, como consecuencia, en uno de los factores con las correlaciones positivas más considerables con los síntomas. Eso significa que, para que un individuo desarrolle las sintomatologías analizadas en el presente estudio, la pareja consiste en uno de los mayores factores de riesgo $\mathrm{y}$, simultáneamente, en el mayor factor protector de ellas. En este sentido, investigaciones como la de Vera-Villarroel, Pavez, Celis-Atenas y Díaz-Pardo (2013) y Sandhya (2009) evidencian que la pareja es una fuente de apoyo que desempeña un papel importante en la estabilidad emocional. Pero, a su vez, puede ser la fuente de mayor malestar por encima de la familia y amigos (Pereira \& Galaz, 2005).

El tercer estresor más significativo para predecir un elevado nivel de síntomas, sobre todo de la depresión, es el conflicto de familia-trabajo. Al analizar más detenidamente esta asociación, se puede encontrar que el efecto del conflicto trabajofamilia sobre conflicto familia-trabajo consiste en uno de los efectos directos más significativos de todo el modelo. Aunque ya Gutek, Searle y Klepa (1991) demostraron la bidireccionalidad del conflicto trabajofamilia y el conflicto familia-trabajo, Netemeyer et al. (1996) destacaron que la mayoría de estudios se concentraba en el análisis del conflicto trabajo-familia. El presente estudio demostró la importancia de diferenciar entre ambos constructos: cuando el conflicto de trabajo-familia tiene las asociaciones positivas más considerables con los otros estresores, el conflicto de familia-trabajo tiene las correlaciones positivas más considerables con los síntomas. Esas características fundamentalmente distintas pueden reconocerse en la Ilustración 3, pues el conflicto de trabajo-familia es la única variable exclusivamente independiente del modelo, quiere decir que es el factor que inicia un determinado proceso. Además, se advierte que los estresores del trabajo reciben un efecto por parte del conflicto trabajo-familia y eso significa que el trabajo en sí no consisten en un estresor de contagio (Bolger et al., 1989; Liu \& Doan, 2020), sino el conflicto trabajo-familia. Por otro lado, el efecto negativo que los conflictos trabajo-familia tienen sobre los estresores de los amigos puede considerarse como inesperado. De acuerdo con estos resultados, un aumento en la percepción de conflictos trabajo-familia conduce hacia una menor percepción de estrés por parte de los amigos. Teniendo en cuenta que el trabajo y la familia se definen como dos entornos principales que no sólo satisfacen diversas demandas humanas, sino que consisten también en importantes fuentes de la felicidad (Zhang, Liu, \& Liu, 2010), podría intuirse que el conflicto trabajo-familia absorbe de alguna manera la atención del individuo $\mathrm{y}$, de esta manera, provoca la percepción disminuida por parte de los estresores de los amigos. Otro resultado inesperado consiste en el efecto positivo que los estresores del trabajo tienen sobre los recursos del trabajo, quiere decir que la aumentada percepción de estresores laborales aumenta también la percepción 
de recursos laborales. Al analizar los ítems correspondientes a ambos factores, se advierte que estos hacen referencia a personas indeterminadas, por lo que la explicación más plausible comprende que, entre más se percibe la existencia de personas que distorsionan las relaciones laborales, más se percibe la existencia de otras personas de confianza que aportan algún tipo de apoyo.

Los hijos como estresores ocupan el tercer lugar con respecto a las variables con asociaciones positivas con los síntomas, pero se observa que este efecto es mayoritariamente sobre la ansiedad. De esta manera, puede destacarse una importante diferencia con respecto al conflicto familia-trabajo, ya que éste tiene mayor efecto sobre la depresión. Este resultado nos conduce hacia la necesidad de diferenciar, por un lado, entre determinados tipos de estresores que pueden surgir en el núcleo familiar y, por otro lado, entre sus respectivas naturalezas, ya que cada uno de ellos predice una sintomatología distinta.

Por último, se analizó la cantidad de efectos que cada factor tiene sobre las demás variables. Estresores hijos es la variable con mayor número de efectos directos $(\mathrm{n}=5)$ : sobre estrategias pandemia, estresores pareja, ansiedad, recursos hijos y recursos trabajo. Si bien los hijos son fuente de satisfacción y apoyo, también contribuyen a aumentar el nivel de estrés de sus padres (Keizer, Dykstra, \& Poortman, 2010; Kiecolt, Blieszner, \& Savla, 2011). De esta manera, consiste en el segundo factor más considerable para reducir la percepción de los recursos

\section{Conclusión}

Durante la pandemia del COVID-19, la pareja como recurso consistió en el principal factor que puede reducir el riesgo de que se desarrollen síntomas depresivos y/o ansiosos. Los estresores de la pandemia y los hijos como estresores son los predictores más considerables de la ansiedad y, por otro lado, los predictores más considerables de la depresión son la pareja como estresor, los conflictos familiatrabajo, los estresores de la pandemia. De esta manera, se concluye que, aunque los estresores de la pandemia están significativamente correlacionados con la depresión y ansiedad, la pareja consiste en la variable clave por sus asociaciones con niveles altos y bajos de los síntomas analizados en el presente estudio.

A pesar de que se ha podido desarrollar un modelo estructural significativamente exacto y que el análisis multigrupo determinó la invarianza factorial por género, deben tenerse en cuenta las siguientes limitaciones: debido al diseño transversal, al muestreo no probabilístico, la poca cantidad de participantes masculinos, el tamaño muestral reducido, la utilización de instrumentos de auto-reporte y de transformaciones de Yeo y Johnson (2000), la generalización de sus resultados resulta dificultosa. Esta afirmación cobra incluso mayor importancia al considerar que (1) se realizó una medida de purificación de los instrumentos psicométricos, que (2) se utilizó el método Backwards Elimination para la selección de variables y que (3) se ajustó el modelo a través de los índices de modificación. De esta manera, cabe 
destacar que el modelo se desarrolló a partir de un enfoque exploratorio (bottomup), el cual conlleva también un determinado riesgo de que los resultados estén sesgados y que sean idiosincráticos

\section{Referencias}

Altena, E., Baglioni, C., Espie, C. A., Ellis, J., Gavriloff, D., Holzinger, B., ... Riemann, D. (2020). Dealing with sleep problems during home confinement due to the COVID-19 outbreak:

Practical recommendations from a task force of the European CBT-I Academy. Journal of Sleep Research, 29(4), 170-173.

https://doi.org/10.1111/jsr.13052

Bellavia, G. M., \& Frone, M. K. (2005). WorkFamily Conflict. In J. Barling, E. K. Kelloway, \& M. R. Frone (Eds.), Handbook of Work Stress (pp. 113148). SAGE Publications, Inc. https://doi.org/10.4135/9781412 975995.n6

Billings, A. G., \& Moos, R. H. (1981). The role of coping responses and social resources in attenuating the stress of life events. Journal of Behavioral Medicine, 4(2), 139-157. https://doi.org/10.1007/BF00844 267

Bolger, N., DeLongis, A., Kessler, R. C., \& Wethington, E. (1989). The Contagion of Stress across Multiple Roles. Journal of Marriage and the Family, 51(1), 175-183. https://doi.org/10.2307/352378 con la muestra. Estas circunstancias nos conducen hacia la necesidad de futuros estudios para validar las asociaciones encontradas.

Byrne, B. M. (2016). Structural Equation Modeling with AMOS. Routledge. https://doi.org/10.4324/9781315 757421

Caccia, P. A., De Grandis, M. C., \& Pérez, G. (n.d.). Somatizaciones y Apoyo Social Funcional percibido en el contexto de Aislamiento Social Preventivo y Obligatorio por Covid19, en Buenos Aires, Argentina. Revista de Psicología UNEMI.

Caccia, P. A., De Grandis, M. C., \& Schetsche, C. (2021). Validación del Inventario SISCO del Estrés de Pandemia (ISEP) en población de Buenos Aires, Argentina. PSIENCIA. Revista Latinoamericana de Ciencia Psicológica, 13(1), 28-40. Retrieved from

http://www.psiencia.com/index.ph $\mathrm{p} /$ revista/article/view/1

Chen, F. F. (2007). Sensitivity of Goodness of Fit Indexes to Lack of Measurement Invariance. Structural Equation Modeling: A Multidisciplinary Journal, 14(3), 464-504.

https://doi.org/10.1080/1070551 0701301834

Core Team. (2020). R: A language and environment for statistical 
computing. Vienna, Austria. https://www.r-project.org/

Costello, A., \& Osborne, J. (2005). Best practices in exploratory factor analysis: Four recommendations for getting the most from your analysis. Practical Assessment, Research, and Evaluation, 10(7), 1-9. https://doi.org/10.7275/jyj1-4868

Daniels, D., \& Moos, R. H. (1990). Assessing Life Stressors and Social Resources among Adolescents. Journal of Adolescent Research, 5(3), 268-289. https://doi.org/10.1177/0743554 89053002

Dierdorff, E. C., \& Ellington, J. K. (2008). It's the nature of the work: Examining behavior-based sources of workfamily conflict across occupations. Journal of Applied Psychology, 93(4), 883-892. https://doi.org/10.1037/00219010.93.4.883

Dunn, T. J., Baguley, T., \& Brunsden, V. (2014). From alpha to omega: A practical solution to the pervasive problem of internal consistency estimation. British Journal of Psychology, 105(3), 399-412. https://doi.org/10.1111/bjop.120 46

Fabrigar, L. R., Wegener, D. T., MacCallum, R. C., \& Strahan, E. J. (1999). Evaluating the use of exploratory factor analysis in psychological research. Psychological Methods, 4(3), 272299. https://doi.org/10.1037/1082-

989X.4.3.272

Fu, C. K., \& Shaffer, M. A. (2001). The tug of work and family. Personnel Review, $30(5)$,

502-522.

https://doi.org/10.1108/EUM0000 000005936

Girmay, M., \& Singh, G. K. (2019). Social Isolation, Loneliness, and Mental and Emotional Well-being among International Students in the United States. International Journal of Translational Medical Research and Public Health, 3(2), 75-82. https://doi.org/10.21106/ijtmrph. 82

Grandey, A. A., \& Cropanzano, R. (1999). The Conservation of Resources Model Applied to Work-Family Conflict and Strain. Journal of Vocational Behavior, 54(2), 350370.

https://doi.org/10.1006/jvbe.1998 .1666

Grzywacz, J. G., \& Bass, B. L. (2003). Work, Family, and Mental Health: Testing Different Models of Work-Family Fit. Journal of Marriage and Family, 65(1), 248-261. https://doi.org/10.1111/j.17413737.2003.00248.x

Gutek, B. A., Searle, S., \& Klepa, L. (1991). Rational versus gender role explanations for work-family conflict. Journal of Applied Psychology, 76(4), 560-568. https://doi.org/10.1037/00219010.76 .4 .560 
Heinze, G., Wallisch, C., \& Dunkler, D. (2018). Variable selection - A review and recommendations for the practicing statistician. Biometrical Journal, 60(3), 431449.

https://doi.org/10.1002/bimj.201 700067

Hinton, P., McMurray, I., \& Brownlow, C. (2014). SPSS Explained (2nd ed.). New York: Routledge. https://doi.org/10.4324/9781315 797298

Hofmann, R. J. (1978). Complexity And Simplicity As Objective Indices Descriptive of Factor Solutions. Multivariate Behavioral Research, 13(2), 247-250. https://doi.org/10.1207/s153279 06mbr1302_9

Hoyle, R. H., \& Gottfredson, N. C. (2015). Sample Size Considerations in Prevention Research Applications of Multilevel Modeling and Structural Equation Modeling. Prevention Science, 16(7), 987-996. https://doi.org/10.1007/s11121014-0489-8

Hu, L., \& Bentler, P. M. (1999). Cutoff criteria for fit indexes in covariance structure analysis: Conventional criteria versus new alternatives. Structural Equation Modeling: A Multidisciplinary Journal, 6(1), 155.

https://doi.org/10.1080/1070551 9909540118
Huarcaya-Victoria, J. (2020). Consideraciones sobre la salud mental en la pandemia de COVID19. Revista Peruana de Medicina Experimental y Salud Pública, 37(2), 327-340.

https://doi.org/10.17843/rpmesp. 2020.372 .5419

Huremović, D. (2019). Psychiatry of Pandemics (D. Huremović, Ed.). Cham: Springer International Publishing. https://doi.org/10.1007/978-3030-15346-5

Johnson, M. C., Saletti-Cuesta, L., \& Tumas, N. (2020). Emociones, preocupaciones y reflexiones frente a la pandemia del COVID-19 en Argentina. Ciência \& Saúde Coletiva, 25(suppl 1), 2447-2456. https://doi.org/10.1590/141381232020256.1.10472020

Keizer, R., Dykstra, P. A., \& Poortman, A.-R. (2010). The transition to parenthood and well-being: The impact of partner status and work hour transitions. Journal of Family Psychology, 24(4), 429-438. https://doi.org/10.1037/a002041 4

Kiecolt, K. J., Blieszner, R., \& Savla, J. (2011). Long-Term Influences of Intergenerational Ambivalence on Midlife Parents' Psychological Wellbeing. Journal of Marriage and Family, $\quad 73(2), \quad 369-382$. https://doi.org/10.1111/j.17413737.2010.00812.x 
Kline, R. B. (2015). Principles and practice of structural equation modeling (4th ed.). The Guilford Press.

Korkmaz, S., Goksuluk, D., \& Zararsiz, G. (2014). MVN: An R Package for Assessing Multivariate Normality. The $R$ Journal, 6(2), 151-162. https://doi.org/10.32614/RJ2014-031

Liu, C. H., \& Doan, S. N. (2020). Psychosocial Stress Contagion in Children and Families During the COVID-19 Pandemic. Clinical Pediatrics, 59(910), 853-855. https://doi.org/10.1177/0009922 820927044

Luk, D. M., \& Shaffer, M. A. (2005). Work and family domain stressors and support: Within- and cross-domain influences on work-family conflict. Journal of Occupational and Organizational Psychology, 78(4), 489-508.

https://doi.org/10.1348/0963179 $05 \times 26741$

Macías, A. B. (2020). El estrés de pandemia (COVID 19) en población mexicana (P. E. Ceceñas Torrero, Ed.). Durango: Centro de Estudios Clínica e Investigación Psicoanalítica S.C.

Mardia, K. V. (1970). Measures of multivariate skewness and kurtosis with applications. Biometrika, 57(3), 519-530. https://doi.org/10.1093/biomet/5 7.3.519

Marsh, H. W., Hau, K.-T., \& Wen, Z. (2004). In Search of Golden Rules: Comment on Hypothesis-Testing Approaches to Setting Cutoff Values for Fit Indexes and Dangers in Overgeneralizing $\mathrm{Hu}$ and Bentler's (1999) Findings. Structural Equation Modeling: A Multidisciplinary Journal, 11(3), 320-341.

https://doi.org/10.1207/s153280 07sem1103_2

Martín, F. A., \& Fernández, M. P. (1978). "Path" análisis, modelos de ecuaciones estructurales y variables no observadas. Reis, 3(3), 187-208. https://doi.org/10.2307/4018271 5

Mikulic, I. M. (1998). La evaluación psicológica de los recursos sociales y los estresores de vida. Aportes del inventario LISRES. Sainte Claire Editora.

Netemeyer, R. G., Boles, J. S., \& McMurrian, R. (1996). Development and validation of work-family conflict and family-work conflict scales. Journal of Applied Psychology, 81(4), 400-410. https://doi.org/10.1037/00219010.81.4.400

Nomaguchi, K. M. (2009). Change in WorkFamily Conflict Among Employed Parents Between 1977 and 1997. Journal of Marriage and Family, 71(1), 15-32. https://doi.org/10.1111/j.17413737.2008.00577.x

OMS. (2019). Nuevo coronavirus 2019. Organización Mundial de la Salud. 
https://www.who.int/es/emergen cies/diseases/novel-coronavirus2019

Parasuraman, S., \& Simmers, C. A. (2001). Type of employment, work-family conflict and well-being: a comparative study. Journal of Organizational Behavior, 22(5), 551-568.

https://doi.org/10.1002/job.102

Pereira, M. G. S., \& Galaz, M. M. F. (2005). Estrés, respuestas de afrontamiento e ideación suicida en adolescentes. Psicología y Salud, 15(2), 221-230.

Peterson, R. A., \& Cavanaugh, J. E. (2020). Ordered quantile normalization: a semiparametric transformation built for the cross-validation era. Journal of Applied Statistics, 47(1315), 2312-2327. https://doi.org/10.1080/0266476 3.2019 .1630372

Ramírez-Ortiz, J., Castro-Quintero, D., Lerma-Córdoba, C., Yela-Ceballos, F., \& Escobar-Córdoba, F. (2020). Mental health consequences of the COVID-19 pandemic associated with social isolation. Colombian Journal of Anesthesiology, 48(4), 28.

https://doi.org/10.5554/2256208 7.e930

Raykov, T., \& Marcoulides, G. A. (2006). A first course in structural equation modeling (2nd ed.). Lawrence Erlbaum Associates.

Revelle, W. (2019). psych: Procedures for personality and psychological research. https://cran.rproject.org $/$ package $=$ psych Version $=1.9 .12$

Rosseel, Y. (2012). lavaan: An R Package for Structural Equation Modeling. Journal of Statistical Software, 48(2), 1-36.

https://doi.org/10.18637/jss.v048. i02

Sandhya, S. (2009). The Social Context of Marital Happiness in Urban Indian Couples: Interplay of Intimacy and Conflict. Journal of Marital and Family Therapy, 35(1), 74-96. https://doi.org/10.1111/j.17520606.2008.00103.x

Sandín, B., Valiente, R. M., Chorot, P., Santed, M. A., \& Lostao, L. (2008). SA-45: forma abreviada del SCL-90. Psicothema, 20(2), 290-296.

Saris, W. E., Satorra, A., \& van der Veld, W. M. (2009). Testing Structural Equation Models or Detection of Misspecifications? Structural Equation Modeling: A Multidisciplinary Journal, 16(4), 561-582.

https://doi.org/10.1080/1070551 0903203433

Schetsche, C., Caccia, P. A., Simaes, A. C., Mancini, N. A., Gómez, F. N., Kusmuk, M. N., ... Elgier, A. M. (n.d.). Traducción y validación de las Work-Family Conflict and FamilyWork Conflict Scales.

Shigemura, J., Ursano, R. J., Morganstein, J. C., Kurosawa, M., \& Benedek, D. M. (2020). Public responses to the 
novel 2019 coronavirus (2019nCoV) in Japan: Mental health consequences and target populations. Psychiatry and Clinical Neurosciences, 74(4), 281-282. https://doi.org/10.1111/pcn.1298 8

Spinelli, M., Lionetti, F., Setti, A., \& Fasolo, M. (2020). Parenting Stress During the COVID-19 Outbreak: Socioeconomic and Environmental Risk Factors and Implications for Children Emotion Regulation. Family Process, $1-15$.

https://doi.org/10.1111/famp.126 01

Urzúa, A., Vera-Villarroel, P., Caqueo-Urízar, A., \& Polanco-Carrasco, R. (2020). La Psicología en la prevención y manejo del COVID-19. Aportes desde la evidencia inicial. Terapia Psicológica, 38(1), 103-118. https://doi.org/10.4067/S071848082020000100103

Vera-Villarroel, P., Pavez, P., Celis-Atenas, K., \& Díaz-Pardo, N. (2013). Fuentes de apoyo emocional y su relación con el bienestar subjetivo: Un análisis preliminar. Revista Mexicana de Psicología, 30(2), 115122.

Yeo, I.-K., \& Johnson, R. A. (2000). A new family of power transformations to improve normality or symmetry. Biometrika, 87(4), 954-959. https://doi.org/10.1093/biomet/8 7.4 .954

Yu, C.-Y. (2002). Evaluating cutoff criteria of model fit indices for latent variable models with binary and continuous outcomes. University of California, Los Angeles Los Angeles, CA.

Zhang, J., Liu, Y., \& Liu, Y. (2010). Antecedents of Work-Family Conflict: Review and Prospect. International Journal of Business and Management, 6(1), 98-103. https://doi.org/10.5539/ijbm.v6n $1 \mathrm{p} 89$. 